\title{
Tras la naturaleza del derecho. Reflexiones sobre el desafío de Shapiro*
}

\author{
Looking for the Nature of Law: On Shapiro's Challenge
}

Damiano Canale ${ }^{* *}$

Recepción y evaluación de propuesta: 24/10/2017

Aceptación: 26/02/2018

Recepción y aceptación final: 16/08/2018

Resumen: Este ensayo se enfoca críticamente en los aspectos metodológicos del libro Legalidad de Scott Shapiro. De hecho, el libro de Shapiro presenta varias tesis originales sobre la naturaleza del derecho y los principales problemas de la filosofía del derecho, pero también sobre cómo la filosofía del derecho puede descubrir la naturaleza del derecho. En este sentido, el método de investigación adoptado por Shapiro puede considerarse como uno de los resultados más interesantes y desafiantes de su investigación. El ensayo está dividido en dos partes. La primera proporciona un análisis del enfoque filosófico de Shapiro; en particular, se centra en el recurso de Shapiro al vocabulario metafísico, el análisis conceptual, el razonamiento constructivo y la explicación institucional del derecho. La segunda parte destaca algunos de los problemas que plantea este enfoque. En particular, el ensayo argumenta que (1) la teoría del derecho de la planificación no puede explicar la obligación legal; (2) la estrategia constructiva de Shapiro tiene un carácter recursivo que tiende a oscurecer la variedad de entidades legales; (3) la versión de análisis conceptual propuesta en Legalidad es semánticamente ciega y corre el riesgo de volver a leer en el mundo las características del lenguaje; (4) Shapiro supone que las entidades

* Traducción del inglés de Federico José Arena.

** Profesor de Filosofía del derecho, Università Bocconi, Milán.

E-mail: damiano.canale@unibocconi.it 


\section{Damiano Canale}

legales equivalen a un solo universo de hechos legales, mientras que las normas, contratos, parlamentos, etc., reales no parecen tener el mismo conjunto básico de propiedades y existir de la misma manera. Para dar cuenta de esto, el ensayo describe una visión alternativa sobre la naturaleza del derecho basada en un enfoque pluralista de la ontología social. Palabras clave: filosofía del derecho, naturaleza del derecho, ontología social.

Abstract: This essay critically focuses on the methodological aspects of Scott Shapiro's book Legalidad. Indeed, Shapiro's book sets out several original theses about not only the nature of law and the main problems of jurisprudence, but also about how the nature of law can be discovered by jurisprudence. In this sense, the method of inquiry adopted by Shapiro can be considered as one of the most interesting' and challenging outcomes of his research. The essay is divided into two parts. The first one provides an analysis of Shapiro's jurisprudential approach; in particular, it focuses on Shapiro's resort to metaphysical vocabulary, conceptual analysis, constructive reasoning, and institutional explanation of law. The second part highlights some of the problems that this approach gives rise to. In particular, the essay argues that (1) the planning theory of law is not able to explain legal obligation; (2) Shapiro's constructive strategy has a recursive character which tends to obscure the variety of legal entities; (3) the version of conceptual analysis proposed in Legality is semantically blind and runs the risk of reading back into the world the features of language; (4) legal entities are supposed by Shapiro to amount to a single universe of legal facts, whereas actual norms, contracts, parliaments, etc. do not seem to have the same basic set of properties and to exist in the same way. To account for this, the essay outlines an alternative view on the nature of law based upon a pluralistic approach to social ontology.

Keywords: jurisprudence, nature of law, social ontology. 
Tras la naturaleza del derecho. Reflexiones sobre...

\section{Pegar las figuras donde corresponda}

Imagine un libro de juegos para niños diseñado de la siguiente manera. Del lado derecho de cada página hay dibujada una escena compleja como, por ejemplo, animales en una selva, Tomás conduciendo una bicicleta amarilla, mamá en el supermercado chino, etc. Del lado izquierdo de la página hay un conjunto de figuras autoadhesivas: el elefante, el sombrero de Tomás, la tetera, etc. El juego consiste en que la niña debe encontrar en la escena de la derecha el objeto que se corresponde con cada uno de los autoadhesivos. Imagine ahora un segundo libro de juegos para niños, con un diseño similar, pero en el que del lado derecho de cada página hay dibujado solo el fondo de una escena como, por ejemplo, un campo de hierba verde, unas montañas que recortan el cielo, una cocina vacía. La tarea de la niña en este segundo caso es diferente, puesto que ahora lo que tiene que hacer es componer su propia escena usando los autoadhesivos, de modo tal que la composición se corresponda con el fondo propuesto. La niña juega con éxito el primer tipo de juego cuando cada figura ha sido pegada en el lugar correcto. La niña juega con éxito el segundo juego cuando la composición que ha creado sobre el fondo propuesto es lo más significativa posible; en este caso, si la niña tiene éxito, las figuras también estarán en el lugar correcto, pero en un sentido diferente.

Ahora bien, piense el lado derecho de estos libros como si fuera el mundo del derecho, compuesto de leyes, precedentes, contratos, testamentos, jueces, parlamentos, etc., y piense en las figuras autoadhesivas como en un conjunto de enunciados que consideramos verdaderos acerca de ese mundo. Respecto de cada enunciado podemos preguntarnos "¿qué lo hace verdadero?". Cuál sea el significado de la expresión entrecomillada dependerá, claramente, del juego. Si el objetivo del juego es dar cuenta del mundo del derecho sin alterarlo, entonces para poder jugar debemos saber qué hechos del mundo jurídico tienen la forma justa. Si el juego tiene por objetivo dar una nueva forma al mundo jurídico, entonces debemos decidir qué forma ha de asumir el mundo jurídico para que tenga sentido dado el trasfondo existente. 


\section{Damiano Canale}

Los filósofos del derecho que van tras la naturaleza del derecho se parecen bastante a una niña que pega figuras en un libro de juegos. Algunos de ellos parecen jugar el primer tipo de juego, es decir, practican la filosofía del derecho descriptiva e intentan dar cuenta del derecho tal como es. Otros prefieren el segundo tipo de empresa, y en este caso practican la filosofía del derecho prescriptiva y nos dicen cómo debería ser el derecho. Sin embargo, como bien sabe cualquier niña, lograr pegar las figuras en el lugar correcto puede ser una tarea bien difícil, en cualquiera de los dos juegos. La dificultad del juego depende de las exigencias que se impongan a los jugadores, tales como la complejidad de la escena, la forma de los autoadhesivos y, obviamente, depende de la habilidad del jugador. La metodología filosófico-jurídica se concentra en determinar cómo llevar a cabo esta tarea, identificando las condiciones de éxito del juego filosófico-jurídico, como también cuáles son sus reglas, y de qué manera se cumple mejor con ellas.

En su libro Legalidad, Scott Shapiro propone una original estrategia para, en filosofía del derecho, pegar los autoadhesivos en el lugar correcto. La estrategia que propone es un tipo de filosofía del derecho descriptiva, que Shapiro usa como dispositivo metodológico para resolver los principales problemas de la filosofía del derecho. De hecho, como introducción al libro de Shapiro bien podrían recordarse las palabras que Lon Fuller usó en su famoso comentario sobre El concepto de derecho de Hart:

[Este libro] es seguramente una contribución a la literatura filosófico-jurídica de un tipo que no contábamos desde hacía mucho tiempo. No se trata de una colección improvisada de ensayos, reunidos en un libro. No es un manual, en el sentido usual de ese término. Se trata, en cambio, de un intento por presentar de modo breve las soluciones que el autor propone para los principales problemas de la filosofía del derecho ${ }^{1}$.

1 Fuller, L. L., The Morality of Law, revised edition, New Haven-London, Yale University Press, 1969, p. 133. 
Tras la naturaleza del derecho. Reflexiones sobre...

Al igual que el libro revolucionario de Hart, Legalidad no tiene la pretensión de aclarar una vieja historia, ni tampoco ha sido concebido como un capítulo ulterior de una novela escrita por otros. Su propósito es contar una historia que nadie ha contado antes, una historia que pretende cambiar el modo en que la filosofía del derecho ha comprendido la naturaleza del derecho. Comenzando por el problema del origen del derecho y de la autoridad jurídica, denominado "paradoja de la autoridad", Shapiro analiza críticamente las soluciones que el positivismo jurídico tradicional ha propuesto y sostiene que son defectuosas $^{2}$. Bentham, Austin, Kelsen y Hart no han logrado explicar cómo el derecho puede generar obligaciones jurídicas sobre la mera base de hechos sociales. Continuando con la tradición positivista, pero también reformulando los problemas que han constituido el objeto principal de esa tradición hasta la actualidad, Shapiro propone su propia solución a la "paradoja de la autoridad", a saber, el derecho es una actividad de planificación que coordina nuestro comportamiento con el objetivo de resolver dudas y desacuerdos acerca de cuestiones morales que afectan nuestra vida en comunidad. La conquista de ciertas metas morales, tales como el mantenimiento del orden social, la prevención de comportamientos desviados, la protección de derechos, la solución equitativa de las controversias, etc., se ve por lo general obstaculizada a causa de la complejidad y la conflictividad de la vida social, o al menos eso es lo que sostiene Shapiro. De acuerdo con la teoría del derecho como plan, "el principal cometido del derecho es precisamente resolver esas

2 El modo en el que Shapiro diseña la paradoja de la autoridad-¿cómo y por qué el derecho puede imponer deberes sobre la sola base de hechos sociales?- resulta fuertemente influenciado por el análisis que Mark Greenberg hace del mismo problema. Según Greenberg los hechos no-normativos no pueden en sí mismos determinar de manera constitutiva el contenido de las leyes. Véase Greenberg, M., "How Facts Make Law", en Hershovitz, S. (ed.), Exploring Law's Empire: The Jurisprudence of Ronald Dworkin, Oxford, Oxford University Press, 2006. El diseño teórico de la paradoja de la autoridad repercute fuertemente en el resto del libro; específicamente, en la lectura que Shapiro hace de la tradición del positivismo jurídico, en el modo en que son concebidos los desacuerdos morales y en la idea según la cual la planificación social es una actividad institucional que posee una finalidad moral. En este artículo, sin embargo, no me concentraré en esta cuestión. 
cuestiones"3. Las instituciones jurídicas resuelven dudas y desacuerdos morales mediante la planificación social. Así, las normas jurídicas son planes sociales que distribuyen derechos y asignan responsabilidades de modo tal que "el ejercicio de las facultades conferidas y la observancia de los deberes asignados alcanza los objetivos seleccionados y

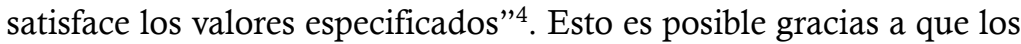
planes, vistos como hechos sociales, alientan intrínsecamente a quienes los comparten a conformarse a ellos. Quienes adoptan un plan están racionalmente obligados a llevarlo a cabo sin que esto se vea afectado por el contenido del plan o sin que se vea influenciado por cursos de acción conflictivos ${ }^{5}$.

Sobre la base de este esquema teórico, en la segunda parte del libro, Shapiro reconstruye críticamente, de un modo admirablemente claro, algunos de los debates filosóficos relativos a las tesis principales del positivismo jurídico. En particular, ofrece un interesante análisis de los rasgos de la interpretación jurídica y proporciona una explicación del modo en que la confianza y la desconfianza impactan en el diseño institucional de un sistema.

Por supuesto, una empresa filosófica de esta envergadura y espesor conlleva seguramente algunos riesgos. En primer lugar, probablemente provocará reacciones críticas de parte de quienes no reconozcan en la teoría de Shapiro ninguna de las tesis tradicionales o de parte de quienes no vean ninguna ventaja en el hecho de considerar las leyes como planes. En segundo lugar, Shapiro pone todos los huevos en una única canasta. Es decir, si luego de un examen riguroso las tesis centrales presentadas en el libro resultan ser equivocadas, o de poco interés para los juristas y filósofos, la empresa filosófica de Shapiro fracasará totalmente. Si, por el contrario, las soluciones de los principales problemas de la filosofía del derecho propuestas en Legalidad fueran plausibles e iluminadoras para nuestra comprensión general

3 Shapiro, S., Legalidad, Madrid-Barcelona, Marcial Pons, 2014, p. 375.

4 Ídem.

265 Véase la sección 6 más abajo. 
Tras la naturaleza del derecho. Reflexiones sobre...

del derecho, entonces la contribución de Shapiro produciría avances extremamente relevantes.

En este trabajo no ofreceré una lectura crítica de Legalidad ${ }^{6}$. Me concentraré en cambio en los aspectos metodológicos de su trabajo, tal como son presentados principalmente en el primer capítulo del libro. En efecto, Legalidad contiene numerosas tesis originales referidas a la naturaleza del derecho, al modo en que la naturaleza del derecho puede ser conocida "pegando los autoadhesivos donde corresponde". En este sentido, el método filosófico adoptado por Shapiro puede bien ser considerado uno de los resultados más interesantes y desafiantes de su investigación filosófica. El análisis que llevaré a cabo del enfoque de Shapiro mostrará, en particular, que la teoría del derecho como plan se caracteriza por un "sobrecompromiso" que tiende a reducir su potencial explicativo. Shapiro intenta no solo descubrir la esencia del derecho, sino también explicar, a través de ella, todos y cada uno de los hechos e instituciones jurídicas, asumiendo que todos los aspectos de la realidad jurídica poseen las mismas condiciones de identidad e iguales propiedades necesarias. Intentaré demostrar que un compromiso de este tipo no es ni necesario ni útil para explicar la naturaleza del derecho. Legalidad podría ser una contribución valiosa para la filosofía del derecho incluso si Shapiro no hubiera asumido un determinado compromiso respecto de la naturaleza del derecho y, en cambio, hubiera hecho espacio a una perspectiva pluralista.

Este artículo se encuentra dividido en dos partes. En las primeras dos secciones describiré el método filosófico de Shapiro concentrándome en el uso de vocabulario metafísico, del análisis conceptual, del razonamiento constructivo y de la explicación institucional del derecho. En las secciones siguientes consideraré algunos de los problemas de este enfoque y sugeriré algunos caminos para evitarlos.

6 Una lectura esclarecedora del libro puede encontrarse en Schauer, F., "The Best Laid Plan", Yale Law Journal, 120, 2011, pp. 586-621. 


\section{La naturaleza del derecho reconsiderada}

Según Shapiro, la filosofía del derecho analítica se preocupa por los fundamentos metafísicos del derecho:

La teoría normativa del derecho se ocupa de los fundamentos morales del derecho, mientras que la teoría analítica del derecho examina sus fundamentos metafisicos. [...] [Ella] analiza la naturaleza del derecho y las entidades jurídicas, y su objeto de estudio abarca, entre otros, los sistemas jurídicos, las leyes, las reglas, los derechos; la autoridad, la validez, la obligación, la interpretación, la soberanía, los tribunales, la causa próxima, la propiedad, el delito, el ilícito extracontractual y la negligencia ${ }^{7}$.

Pero ¿qué es exactamente lo que buscamos cuando vamos tras los fundamentos metafísicos del derecho? Típicamente, una investigación metafísica no se concentra en las características contingentes de la realidad, sino que intenta revelar las propiedades necesarias del mundo, aquellas propiedades que no dependen de lo que, de manera contingente, conocemos, queremos o deseamos. En particular, para Shapiro, son dos las cuestiones metafísicas que están aquí en juego: qué es lo que hace que una entidad jurídica sea lo que es (la pregunta de la identidad) y qué consecuencias necesarias se siguen del hecho que esa entidad sea lo que es (la pregunta de la implicación). Una respuesta correcta a la pregunta de la identidad "debe exponer un conjunto de propiedades que hacen de las instancias (reales o posibles) de X el objeto que son" 8 . Además, para responder a la pregunta de la implicación, es necesario descubrir las propiedades de las que el derecho, cualquiera sea su contenido, no puede carecer ${ }^{9}$.

Esta concepción de la filosofía analítica del derecho es bastante similar al modo en que Joseph Raz la concibe y da cuenta de la mayor

7 Shapiro, S., op. cit., pp. 26-27.

8 Ibidem, p. 34.

2899 Ibidem, p. 35. 
Tras la naturaleza del derecho. Reflexiones sobre...

parte de las investigaciones contemporáneas acerca de la naturaleza del derecho ${ }^{10}$. A pesar de ello, en el caso de Shapiro, la referencia explícita al vocabulario metafísico podría resultar un tanto sorprendente. Definitivamente ello es así para aquellos filósofos del derecho, particularmente en el mundo del Civil Law, que todavía conciben la tradición analítica en filosofía como, entre otras cosas, una batalla contra la metafísica que ha de ser llevada adelante mediante las herramientas del análisis conceptual. De hecho, esta batalla ha sido generalmente considerada, en el pasado, como el modo para establecer la distinción fundamental entre positivismo jurídico y teoría del derecho natural, respecto tanto de sus tesis sustanciales como metodológicas. ¿A qué se debía ello?

Según la antigua tradición del positivismo jurídico, la palabra "metafísica" denota aquellos lenguajes jurídicos oscuros, engañosos e incluso sin sentido. Este es típicamente el caso de aquellas tesis acerca de la naturaleza del derecho cuyas condiciones de verdad no son ni empíricas ni conceptuales. Si se afirma, tal como diría un defensor del derecho natural, que la verdadera fuente de la legalidad es la moral, no es fácil especificar cuáles son las condiciones bajo las cuáles esta afirmación metafísica es verdadera o falsa. Ello en cuanto tales condiciones no parecen depender ni de hechos empíricos ni del significado de los términos usados. Si esto es así, la afirmación en cuestión no se conecta con nada en la realidad y la metafísica se transforma entonces en un sofisticado truco que hace del derecho "una solemne omnipotencia en el cielo"11. En consecuencia, la filosofía del derecho

${ }^{10}$ Véase Raz, J., Between Authority and Interpretation: On the Theory of Law and Practical Reason, Oxford, Oxford University Press, 2009, pp. 17-46, y Raz, J., Ethics in the Public Domain: Essays on the Morality of Law and Politics, Oxford, Clarendon Press, 1994, pp. 195-209. Véase también Dickson, J., Evaluation and Legal Theory, Oxford-Portland, Hart, 2001, p. 17.

11 Justice Holmes discrepando en Southern P. Co. v. Jensen, 244 U.S. 205, 222 (1917). Véase también Kelsen, H., General Theory of Law and State, Cambridge (Mass.), Harvard University Press, 1945, p. 433. Alguien podría señalar que Jeremy Bentham usaba frecuentemente la palabra "metafísica" en sentido positivo; pero en realidad Bentham en esos casos usaba el término para denotar un discurso que consiste en "explicar o investigar qué es lo que otra persona quiere decir" (Bentham, J., Rationale of Judicial 
ha de encargarse de desmitificar, mediante el análisis conceptual, las explicaciones del derecho que recurran a un vocabulario metafísico.

Es necesario notar que este cuadro es erróneo. En primer lugar, los filósofos del derecho natural contemporáneos tales como Lon Fuller y John Finnis han intentado separar la investigación acerca de la naturaleza del derecho de consideraciones metafísicas. Han concentrado su investigación filosófico-jurídica en las propiedades de la razón práctica, preguntándose si estas propiedades poseen cierta continuidad con el derecho ${ }^{12}$. Asimismo, el supuesto compromiso antimetafísico de la filosofía del derecho analítica se desmorona si se asume -tal como Quine sugiere que deberíamos hacer- que no existe una verdadera distinción entre verdades analíticas y verdades empíricas ${ }^{13}$. Si esto es así, entonces no existen las bases suficientes para oponer enunciados existenciales metafísicos a enunciados existenciales empíricos. La inteligibilidad de los enunciados metafísicos puede obtenerse si se los formula mediante oraciones observacionales ${ }^{14}$. Por ejemplo, la afirmación metafísica "la moral es la verdadera fuente de la legalidad" puede ser aclarada de la

Evidence, London, Hunt \& Clarke, 1827, vol. III, p. 386), i. e., una investigación acerca del significado de los enunciados metafísicos. Respecto de la metafísica tradicional, en cambio, Bentham era mucho más cauto: “'Odio la metafísica' exclama Eduard Burke en algún pasaje, no le faltaban causas para hacerlo" (idem).

12 Véase Finnis, J., Natural Law and Natural Rights, second edition, New York-Oxford, Oxford University Press, 2011; Fuller, op. cit.

${ }^{13}$ Sobre esto, véase Canale, D., "Consequences of Pragmatic Conceptualism. On the Methodology Problem in Jurisprudence", Ratio Juris, 22, 2009, pp. 171-186; Himma, K., "Reconsidering a Dogma: Conceptual Analysis, the Naturalistic Turn, and Legal Philosophy", in Freeman, M. y Harrison, R. (eds.), Law and Philosophy: Current Legal Issues, Oxford, Oxford University Press, 2007; Leiter, B., Naturalizing Jurisprudence. Essays on American Legal Realism and Naturalism in Legal Philosophy, Oxford, Oxford University Press, 2007; Oberdiek, J. y Patterson, D., "Moral Evaluation and Conceptual Analysis in Jurisprudential Methodology", en Freeman, M. y Harrison, R. (eds.), Law and Philosophy: Current Legal Issues, Oxford, Oxford University Press, 2007, pp. 60-75; Coleman, J., "Naturalized Jurisprudence and Naturalized Epistemology", Philosophical Topics, 29, 2001, pp. 113-128.

14 "Si no existe una distinción clara entre analítico y sintético [...], las cuestiones ontológicas terminan ubicándose junto a las cuestiones de la ciencia natural" (Quine, W. V. O., "On Carnap's Views on Ontology", en Id., The Ways of Paradox and Other Essays, New York, Random House, 1966, p. 134). 
Tras la naturaleza del derecho. Reflexiones sobre...

siguiente manera: "Para todo $X$ si $X$ es jurídico entonces $X$ es moral y existe un $Y$ tal que $Y$ es la fuente de $X$ e $Y$ es moral". Este enunciado metafísico es perfectamente inteligible, en el sentido de que puede ser verdadero, tal como lo sostiene la teoría del derecho natural, o falso, tal como la considera el positivismo jurídico. La asunción general de Quine, por lo tanto, parecería reivindicar la investigación metafísica contra el análisis conceptual en filosofía del derecho si bien, tal como será señalado en la próxima sección, este no es el punto de vista de Shapiro.

En realidad, de este modo se produce una modificación radical de la función filosófica del vocabulario metafísico, que no puede ser considerada una buena noticia para la filosofía del derecho que persiga un análisis metafísico de los fundamentos del derecho. Desde el punto de vista de Quine, la metafísica pierde su rol fundacional. Por un lado, los enunciados metafísicos son ahora puramente extensionales y la existencia puede ser atribuida exclusivamente a objetos físicos o a clases abstractas. Por el otro lado, dada ciertas condiciones, diferentes ontologías puede ser igualmente útiles para la teoría del derecho. Un compromiso con un marco metafísico, ya sea en filosofía del derecho como en cualquier otro tipo de discurso teórico, no es nunca absoluto, sino que depende de cuestiones pragmáticas relativas al poder explicativo de la teoría que incluye el marco metafísico en cuestión, y de sus criterios de revisión ${ }^{15}$.

Si esto es así, ¿qué queda para una investigación filosófico-jurídica acerca de la naturaleza del derecho? Desde un punto de vista metodológico, una investigación de ese tipo puede ser concebida como gobernada por una inferencia a la mejor explicación ${ }^{16}$. Los filósofos

${ }^{15}$ Quine, W. V. O., Word and Object, Cambridge (Mass.), MIT Press, 1960, p. 271. Ciertamente, la crítica de Quine a la distinción analítico/sintético podría ser, a su vez, cuestionada reivindicando la autonomía del análisis conceptual, en este sentido véase Himma, op. cit. Alternativamente, la crítica de Quine puede ser incorporada dentro de una versión actualizada del análisis conceptual, tal como lo propone Franck Jackson, quien es la principal fuente de inspiración de Shapiro al respecto. Véase Jackson, F., From Metaphysics to Ethics. A Defense of Conceptual Analysis, Oxford, Clarendon Press, 1998, pp. 44-55.

${ }^{16} \mathrm{La}$ "inferencia a la mejor explicación" coincide aproximadamente con lo que suele denominarse "abducción" o "inferencia hipotética": "Al hacer esta inferencia uno 
del derecho no buscan explicar hechos contingentes acerca de una región espacio-temporal. No están interesados en determinar si $X$ es una norma que pertenece al derecho del estado de Connecticut, o si $Y$ es un contrato válido de acuerdo con el derecho contractual italiano. La filosofía del derecho pretende ofrecer una explicación de algunos hechos necesarios (aquellos hechos sin los cuales el derecho no tendría lugar), a partir de otros hechos necesarios más fundamentales, que instancian las condiciones de identidad y las propiedades necesarias de las entidades jurídicas. Estos hechos necesarios más fundamentales pueden ser vistos como los hechos acerca de la naturaleza del derecho.

Obviamente, el término "naturaleza" es en este contexto un término ambiguo. Puede ser usado para transmitir la idea de que los enunciados iusfilosóficos describen estados de cosas naturales. Desde este punto de vista, por ejemplo, el enunciado metafísico "la moral es la verdadera fuente de la legalidad" puede ser desambiguado como sigue: "para todo hecho legal $Y$, existe un hecho moral $X$ tal que $X$ determina $Y$ ". Pero el término "naturaleza" puede también ser visto como comprometiendo a los filósofos del derecho con un tipo de explicación distinto, tal como implícitamente sostiene Shapiro. Los hechos que forman parte del fundamento del derecho se refieren a aquello que debe ser el caso, y la naturaleza normativa de tales hechos no puede ser parte de un marco explicativo meramente causal. Estos hechos valorativos se refieren a disposiciones humanas que supervienen sobre hechos físicos. La disposición a asumir ciertas actitudes y a realizar ciertas evaluaciones, la disposición a usar estas actitudes para formar intenciones y comportarse conforme a ellas, y la disposición a

infiere, a partir del hecho de que una determinada hipótesis explicaría la evidencia, que la hipótesis es verdadera. Por lo general, existirán numerosas hipótesis que lograrían explicar la evidencia, por lo que uno debería ser capaz de desechar todas esas hipótesis alternativas antes de considerar garantizada la inferencia. Así, se infiere, a partir de la premisa que una determinada hipótesis ofrece una 'mejor' explicación de la evidencia que la que ofrecería cualquier otra hipótesis, la conclusión de que la hipótesis en cuestión es verdadera" (Harman, G. H., "The Inference to the Best Explanation", The Philosophical Review, 74, 1965, p. 89). Este tipo de inferencia describe perfectamente el "trabajo de detective" que, según Shapiro, caracteriza el tipo de análisis conceptual que adopta. Véase Shapiro, op. cit., p. 39. 
Tras la naturaleza del derecho. Reflexiones sobre...

realizar evaluaciones para establecer si lo que hacemos es un comportamiento adecuado o inadecuado. Asimismo, los hechos evaluativos no poseen únicamente propiedades disposicionales sino que también poseen una naturaleza social. Son hechos acerca del modo en el que nuestras intenciones afectan nuestra vida social, condicionan nuestras relaciones con los demás, estructuran el comportamiento social y dan forma a las instituciones sociales ${ }^{17}$. Para Shapiro, estos son los hechos sociales realmente importantes para una investigación sobre la naturaleza del derecho. Sin embargo, desde un punto de vista metodológico, una tesis de este tipo parece conducir la filosofía del derecho hacia uno de los cuernos del siguiente dilema: o bien los hechos señalados arriba han de ser explicados empíricamente, o bien su "explicación" ha de recurrir a un vocabulario normativo. En el primer caso, la filosofía del derecho no será otra cosa más que una ciencia social y un filósofo no tendrá nada para decir que no pueda ser dicho de modo más adecuado por un sociólogo, un psicólogo o un antropólogo. En el segundo caso, una explicación de la naturaleza del derecho expondrá cómo debe ser el derecho y por lo tanto se alejará del positivismo jurídico y de la filosofía del derecho descriptiva.

Shapiro toma un tercer camino, que lo lleva a reformular, al menos parcialmente, el modo tradicional de concebir el análisis conceptual dentro de la filosofía del derecho

${ }^{17}$ La idea según la cual los hechos normativos existen y son necesarios para determinar el contenido del derecho ha dado lugar a profundas controversias. Véase Leiter, $o p$. cit., p. 121 y ss. y Greenberg, M., "Naturalism in Epistemology and the Philosophy of Law", Law \& Philosophy, 30, 2011, pp. 419-451. Esta cuestión es parte de la discusión filosófica acerca de la denominada "normatividad del derecho", es decir, acerca de cómo es el caso que el derecho proporciona a los miembros de la comunidad razones para actuar. Para una reconstrucción crítica de este debate véase Enoch, D., "Reasons-Giving and the Law," en Green, L. y Leiter, B. (eds.), Oxford Studies in Philosophy of Law, vol. 1, Oxford, Oxford University Press, 2011. 


\section{Del análisis conceptual a la filosofía de la acción}

Para Shapiro, las respuestas a la pregunta de la identidad y a la pregunta de la implicación han de ser obtenidas mediante el análisis conceptual. Esta tesis podría incluso parecer más problemática que el compromiso metafísico apenas analizado. De hecho, la filosofía del derecho analítica contemporánea ha recurrido al vocabulario metafísico debido a que su método tradicional (el análisis conceptual) parece haber fracasado. El fracaso del método tradicional se debe precisamente a los argumentos de Quine contra la distinción analítico-sintética. Como hemos visto en la sección precedente, estos argumentos justifican recurrir al vocabulario metafísico. Por lo tanto, ¿cómo es posible que el análisis conceptual pueda servir para descubrir la naturaleza de las entidades jurídicas? Recurrir al análisis conceptual, o bien impide el conocimiento de la naturaleza del derecho, o bien vacía de significado cualquier explicación de ella. La solución a este rompecabezas la provee el tipo de análisis conceptual que Shapiro practica en Legalidad. ¿Cómo lo concibe?

Según Shapiro, el objeto del análisis conceptual no es un concepto sino "la entidad a la que se aplica el concepto"18. En otras palabras, el análisis de conceptos como DERECHO, AUTORIDAD, OBLIGACIÓN, etc., no involucra el significado de las palabras "derecho", "autoridad", "obligación", etc., sino las entidades que instancian ese contenido en la realidad, y por lo tanto pueden ser identificadas como derecho, autoridades, obligaciones, etc. Para hacer esto, el análisis conceptual ha de parecerse al trabajo de un detective.

En el análisis conceptual, el filósofo también recaba pistas y emplea el proceso de eliminación con un propósito específico, concretamente, elucidar la identidad del objeto al que se aplica el concepto en cuestión. La diferencia más importante entre el filósofo y el detective de policía es que las pruebas que recolecta y analiza este último se relacionan con hechos verdaderos, mientras que al primero básicamente le intere- 
Tras la naturaleza del derecho. Reflexiones sobre...

san las verdades obvias. En otras palabras, las pistas filosóficas no son meramente verdaderas, sino además autoevidentes ${ }^{19}$.

En otras palabras, quien se comprometa con el análisis conceptual en la filosofía del derecho tiene, en primer lugar, la tarea de recolectar la mayor cantidad posible de verdades obvias acerca del derecho. A continuación tiene que preguntarse, desde un punto de vista teórico, cómo ha de ser el derecho si es que ha de poseer las propiedades especificadas en la lista de verdades obvias. En los términos del ejemplo usado al inicio de este artículo, la filósofa del derecho ha de "pegar los autoadhesivos en el lugar que corresponda" construyendo una teoría que mejor dé cuenta del conjunto de verdades obvias acerca del derecho.

Claramente esta presentación del análisis conceptual se aleja bastante del modo tradicional, estilo Oxford, de buscar condiciones analíticamente necesarias y suficientes. Se encuentra en cambio mucho más cerca del enfoque metafísico estilo Camberra ofrecido por Frank Jackson $^{20}$. Según este enfoque, el análisis conceptual comienza a partir de nuestras intuiciones acerca de una cosa o evento, formuladas típicamente mediante afirmaciones consideradas obvias. Luego, se esfuerza por identificar las circunstancias cubiertas por esas afirmaciones e intenta demostrar cómo estas afirmaciones se siguen de una descripción o explicación más fundamental. De este modo, el análisis conceptual muestra la concepción implícita asociada a un término conceptual que, a su vez, determina la condición de identidad de aquello a lo que el término se refiere. Obviamente, las intuiciones desempeñan simplemente un "papel provisorio" en la determinación de cómo es el mundo. Es decir, podemos equivocarnos acerca de aquello que es autoevidentemente verdadero. De todos modos, es fuertemente implausible que el conjunto completo de nuestras intuiciones sobre el mundo sean erróneas. Asimismo, es posible que desacordemos acerca de si un enunciado es una afirmación obvia o no. En la mayoría de los casos, sin embargo, el desacuerdo conceptual puede ser resuelto si se compara el poder explicativo de cada una de las teorías en las que

19 Ibidem, p. 39.

${ }^{20}$ Jackson, op. cit. 
las verdades obvias en cuestión están incluidas ${ }^{21}$. Finalmente, puede suceder que pasemos por alto alguna verdad obvia acerca del derecho y las propiedades necesarias a ella conectadas. Para identificar las propiedades esenciales del derecho que permanecen ocultas, el análisis conceptual necesita el apoyo de "estrategias compensatorias" (compensatory strategies). Shapiro menciona cuatro estrategias de este tipo: 1) La comparación de las instituciones jurídicas con otras prácticas sociales similares (comparative strategy). 2) La solución de las paradojas y los rompecabezas filosóficos (puzzle-solving). 3) El examen de la evidencia histórica y antropológica acerca del derecho (anecdotal strategy). 4) La construcción de un sistema jurídico hipotético a partir de una situación no jurídica, con la finalidad de poder determinar lo que resulta necesario para transformar esta situación en derecho (constructivism strategy). Todo esto muestra que el análisis conceptual es un método de investigación imperfecto y falible ${ }^{22}$. Pero ello no lo vuelve inválido

21 "Para decidirse entre las intuiciones sería necesario analizar las teorías de las que forman parte, a fin de observar cuál se acomoda de manera más adecuada al conjunto completo de juicios razonados acerca del derecho" (Shapiro, op. cit., p. 43).

${ }^{22}$ Podría preguntarse si este proceso intelectual puede ser denominado "análisis conceptual". Yo sostengo que no. De hecho, cuando Jackson y Shapiro usan la palabra "concepto" se refieren a usos del lenguaje. En efecto, Jackson admite que "nuestro objeto es realmente la identificación de las posibles situaciones cubiertas por las palabras que usamos para formular nuestras preguntas [...]. Yo uso el término 'concepto' en parte por deferencia hacia la terminología tradicional que habla de análisis conceptual, y en parte para enfatizar que si bien nuestro objeto es la identificación de las diferentes situaciones cubiertas por porciones del lenguaje según los usuarios del lenguaje, o por la comunidad ( folk) en general, está divorciado de consideraciones locales respecto de un lenguaje en particular" (Jackson, op. cit., p. 33). Respecto del término "análisis", me parece que este tipo de proceso intelectual podría ser descripto más apropiadamente como un tipo de explicación. De hecho, Shapiro se concentra no tanto en la pregunta “¿qué es el derecho?”, sino más bien en la pregunta “¿por qué los usuarios del lenguaje poseen esta noción del derecho?”. Solo una respuesta a la segunda pregunta proporciona acceso a la primera cuestión. Si ello es así, Shapiro podría haber denominado su método como "explicación de los usos del lenguaje ordinario" en lugar de "análisis conceptual", si bien claramente la primera etiqueta es mucho menos atractiva para los filósofos del derecho. 
Tras la naturaleza del derecho. Reflexiones sobre...

como método para la filosofía del derecho si se lo integra con otras formas de razonamiento ${ }^{23}$.

En definitiva, la concepción que Shapiro tiene de la metodología de la filosofía del derecho puede ser resumida como sigue. Primero, una investigación acerca de la naturaleza del derecho ha de recoger la mayor cantidad posible de verdades obvias. Por ejemplo, supóngase que $F(T)$ es el conjunto de verdades acerca del derecho consideradas obvias por la mayor parte de los hablantes competentes. Siguiendo a Moore, podríamos denominar estos elementos como el conjunto de "las asunciones de sentido común acerca del derecho". T puede incluir, como afirma Shapiro, "Todos los sistemas jurídicos tienen jueces", "Todo sistema jurídico cuenta con instituciones para cambiar el derecho", "La autoridad jurídica es conferida por reglas jurídicas", "Es posible obedecer el derecho aunque uno no crea que está moralmente obligado a hacerlo", etcétera ${ }^{24}$. Segundo, $F(T)$ ha de ser coherentizado eliminando errores y resolviendo desacuerdos conceptuales; además ha de ser integrado mediante los truismos ocultos $h T$ que pueden ser descubiertos usando estrategias compensatorias. Tercero, el conjunto de tesis resultante $F(T+h T)$ se identifica mediante un enunciado existencial $R$ que corresponde a $F(T+h T)$ tal como: "Existe un $X$ que de manera característica posee instituciones para ser modificado, impone obligaciones, cuya autoridad es otorgada mediante leyes, es obedecido incluso si uno no cree estar moralmente obligado a hacerlo, etc."25. Una vez hecho esto, el análisis conceptual explica bajo qué condiciones

23 Para Shapiro, "no deberíamos tener una confianza excesiva" respecto de las afirmaciones que señalan una propiedad como formando parte de la naturaleza del derecho y deberíamos estar dispuestos a modificar nuestros puntos de vista. Shapiro, op. cit., p. 46.

${ }^{24}$ Shapiro, op. cit., p. 41.

${ }^{25}$ Bajo este esquema, el enunciado existencial $R$ usado para explicar nuestras asunciones de sentido común acerca del derecho debería tener la estructura de un enunciado à la Ramsey, i. e. una fórmula cuantificada existencialmente en la que todos los términos teóricos secundarios de la teoría del derecho deben ser reemplazados por variables vinculadas (véase Ramsey, F., Philosophical Papers, Cambridge, Cambridge University Press, 1990, p. 112 y ss.). Efectivamente, Shapiro parece hacer referencia aquí a la versión modificada de los enunciados de Ramsey que Jackson usa para identificar las propiedades éticas. Cfr. Jackson, op. cit., pp. 140-141. 
la descripción del derecho en $R$ es verdadera. Con relación a nuestro ejemplo el resultado del análisis sería el siguiente:

(1) Si las cosas son de tal y cual modo según la explicación $E$ de $R$, entonces $R$ es verdadero.

De este modo, el relato acerca de la naturaleza del derecho, usando el vocabulario de sentido común de un hablante competente, se "hace verdadero" gracias al relato que usa un vocabulario más fundamen$\mathrm{tal}^{26}$. Este resultado conceptual es falible y revisable pero, desde el punto de vista de Shapiro, el método apenas delineado es la mejor estrategia que los teóricos del derecho tienen a disposición para descubrir los criterios de identidad y las propiedades necesarias del derecho.

El proceso intelectual apenas descripto posee dos aspectos cruciales, estrictamente relacionados entre sí y sobre los que vale la pena concentrarse. En primer lugar, al igual que en todo "trabajo de detective", debe proponerse una hipótesis que pueda ofrecer la mejor explicación $E$ de las asunciones de sentido común acerca del derecho. En segundo lugar, debe identificarse cuál es el vocabulario que encaja mejor con esa explicación. Respecto de la primera cuestión, la hipótesis más básica de Shapiro en Legalidad es que "somos seres que planifican"27. ¿Qué significa esto? Las palabras "plan" y "planificar" se refieren aquí a una propiedad básica de las acciones humanas que ha sido analizada por primera vez en el trabajo filosófico de Michael Bratman. Según Bratman, uno de los aspectos distintivos de la psicología humana es que los seres humanos desean numerosos fines pero se enfrentan a

\footnotetext{
${ }^{26}$ Véase Jackson, op. cit., p. 28.

${ }^{27}$ Shapiro, op. cit., p. 161. Nótese que la afirmación "somos seres que planifican" es hipotética no en el sentido que el análisis conceptual podría mostrar que se trata de una afirmación equivocada. Según Shapiro, esta afirmación se refiere a un hecho psicológico que no requiere ser puesto en cuestión por el análisis. Es la hipótesis a partir de la cual se infiere la explicación de nuestras asunciones de sentido común acerca del derecho.
} 
Tras la naturaleza del derecho. Reflexiones sobre...

una seria escasez de recursos y a la necesidad de coordinación ${ }^{28}$. Los planes estructuran el razonamiento práctico y la deliberación guiando nuestros cursos de acción. En particular, los planes reducen los costos de la deliberación e imponen exigencias de coherencia entre nuestras creencias y nuestros deseos. De hecho, sobre la base del principio de racionalidad instrumental, una vez que un plan de acción ha sido adoptado se impone la exigencia racional de llevarlo a cabo, sin deliberación ulterior. Esto permite a los individuos tomar decisiones racionales en situaciones en las que no tienen tiempo para deliberar, les permite también involucrarse en proyectos complejos y extendidos en el tiempo, les permite coordinar sus actividades y trabajar conjuntamente para alcanzar la misma meta. De este modo, los individuos pueden "lograr bienes y realizar valores que de otra forma serían inalcanzables" 29 . Si bien la idea de que somos criaturas planificadoras se apoya en un conjunto de asunciones psicológicas acerca de cuáles son las propiedades básicas de los seres humanos, la idea en sí no es, en el análisis de Shapiro, una tesis psicológica. Se trata, en cambio, de una tesis conceptual (en el sentido metafísicamente orientado que hemos previamente señalado). Una tesis a partir de la cual puede construirse una explicación plausible de la naturaleza del derecho. Ello es así puesto que la noción de plan nos ofrece la clave para resolver la paradoja de la autoridad, en cuanto, desde el punto de vista de Shapiro, esta noción explica por qué un cierto tipo de hechos sociales genera genuinas obligaciones.

Respecto de la segunda cuestión, Shapiro asume que el vocabulario de la filosofía de la acción es el que mejor encaja con una explicación de la naturaleza del derecho. Shapiro señala aquí que las preguntas "¿qué es el derecho?” y "¿qué son los sistemas jurídicos?” son ambiguas, en el sentido de que pueden referirse tanto a una investigación sobre la naturaleza de las normas jurídicas como a una investigación acerca de la naturaleza de las organizaciones jurídicas. Ahora bien, la teoría del derecho analítica tradicionalmente ha estudiado el fenó-

28 Bratman, M., Intention, Plans, and Practical Reason, Cambridge (Mass.), Harvard University Press, 1987, p. 2 y ss.

${ }^{29}$ Shapiro, op. cit., p. 160. 
meno jurídico "analizando las normas producidas por las organizaciones jurídicas en vez de las organizaciones que las producen" ${ }^{30}$. Sin embargo, el análisis organizacional se ha vuelto una característica predominante en las ciencias sociales y se ha demostrado fructífero para la explicación de los fenómenos sociales. Dado que los sistemas jurídicos "tienen estructuras institucionales distintivas destinadas a conseguir ciertos objetivos políticos", la falta de interés por el análisis organizacional causa sorpresa. De hecho, para Shapiro, preguntarse "por qué los agentes económicos se organizan en empresas en lugar de realizar transacciones independientes en el mercado en forma continua" 31 es un prerrequisito de toda investigación acerca de la naturaleza de las normas jurídicas. Una respuesta a esta pregunta puede ser ofrecida por los filósofos de la acción, quienes en los últimos años han proveído el vocabulario necesario para el análisis de la naturaleza de los grupos y de la acción colectiva. Por lo tanto, esta rama de la filosofía parece poseer los recursos explicativos que hacen falta para dar cuenta de nuestras asunciones de sentido común acerca del derecho.

Sin embargo, el aspecto más original del uso que en Legalidad Shapiro hace del esquema de Jackson es el modo en que se obtiene la mejor explicación de la naturaleza del derecho. Como hemos visto, Shapiro afirma que existen numerosas "estrategias compensatorias" que pueden ayudarnos en el análisis conceptual. Entre ellas, la más importante es la "estrategia constructivista". Cuando usa esta estrategia, el filósofo del derecho "comienza con una situación no-jurídica muy simple y fácil de comprender. Después utiliza este escenario para realizar una comparación con el derecho e intenta imaginar qué sería necesario para transformarlo en un sistema jurídico"32. En particular, mediante esta estrategia, y a partir del hecho que los seres humanos son criaturas planificadoras, Shapiro deduce el modo en el que este hecho afecta la acción individual, el comportamiento de grupos pequeños, la organización de actividades a gran escala, e incluso actividades 
jerárquicamente estructuradas, masivas e institucionalizadas como el derecho. Esta construcción intelectual pretende justificar la afirmación metafísica según la cual "la actividad jurídica es una forma de planificación social [...]. Siguiendo esta idea, las reglas jurídicas son ellas mismas planes generalizados, o normas similares a los planes, emitidas por quienes están autorizados a planificar para otros"33. Sobre la base de una comparación con otras formas de explicación, la teoría de los planes es, de hecho, considerada la mejor explicación de las asunciones de sentido común acerca del derecho. El resultado final del análisis conceptual es por lo tanto el siguiente:

(2) Si el derecho es una actividad de planificación que posee las propiedades $a, b, c, \mathrm{y} d$, entonces $R$ es verdadero, y el derecho necesariamente posee las propiedades $a, b, c$ y $d$.

En efecto, esta afirmación aclara la respuesta que Shapiro da a la pregunta de la identidad: "la existencia del derecho [...] refleja el hecho de que los seres humanos somos seres que planifican, dotados de capacidades cognitivas y volitivas para organizar el comportamiento a lo largo del tiempo y entre las personas a fin de alcanzar metas muy complejas" 34 . En otras palabras, aquello que hace de las leyes el derecho es que son planes, o normas similares a planes, y nada más.

Sobre la base de la reconstrucción precedente, los compromisos metodológicos que asume la teoría del derecho de Shapiro pueden ser resumidos de la siguiente manera:

(a) El compromiso metafisico: la teoría del derecho lleva adelante una investigación metafísica acerca de la naturaleza del derecho, dirigida a descubrir los criterios de identidad y las propiedades necesarias de las entidades jurídicas.

(b) El compromiso con el análisis conceptual: la investigación metafísica en teoría del derecho se apoya en el análisis conceptual, es decir, 
busca explicar los hechos jurídicos necesarios mediante hechos necesarios más fundamentales.

(c) El compromiso con el constructivismo: los hechos necesarios más fundamentales, relativos a la naturaleza del derecho, pueden ser descubiertos mediante una teoría que ofrezca la mejor explicación de las asunciones de sentido común acerca del derecho.

(d) El compromiso con una explicación institucional del derecho: la teoría que mejor da cuenta de la naturaleza del derecho es la teoría de la planificación social acerca de las actividades compartidas institucionalizadas.

Los compromisos metodológicos de Shapiro son especialmente atractivos y cautivadores. Asumen que la pregunta metafísica acerca de la naturaleza del derecho puede ser objeto de análisis conceptual $y$, de este modo, ofrecen una nueva perspectiva para la filosofía del derecho. Esta perspectiva se diferencia tanto de la teoría del derecho naturalizada como del enfoque estilo Oxford para el estudio de conceptos jurídicos. Pero, al mismo tiempo, este método da lugar a algunas dificultades teóricas que serán señaladas y discutidas en la sección siguiente. Comenzaré concentrándome en el compromiso con una explicación institucional del derecho, y luego retrocederé al resto de los compromisos.

\section{Planes y obligaciones jurídicas}

La teoría del derecho como plan es el resultado teórico más importante de Legalidad. Según esta teoría, las normas jurídicas son planes, o normas similares a planes. Más precisamente, las propiedades que poseen las normas jurídicas, según el cuadro delineado por el vocabulario de sentido común, son idénticas a las propiedades que los planes poseen, de acuerdo con el vocabulario explicativo de segundo orden de la teoría de la planificación social.

Vale la pena notar, sin embargo, que la noción de plan fue originalmente introducida por Michael Bratman en respuesta a un problema teórico particular, que no se relacionaba con las instituciones sociales 
Tras la naturaleza del derecho. Reflexiones sobre...

ni con las actividades masivamente compartidas, como el derecho. La noción de plan se vincula al problema que ponen los cursos de acción cuya explicación no queda cubierta por el modelo básico de la intencionalidad, constituido por la combinación creencia-deseo. Se trata de situaciones en las que la consecución de nuestras metas requiere, típicamente, un tipo de guía y coordinación a lo largo de un espacio prolongado de tiempo, que nuestras creencias y nuestros deseos ordinarios no parecen ofrecer. Los planes, en cambio, permiten explicar este tipo de intencionalidad que implica un particular compromiso con acciones futuras. Es decir, un tipo de intencionalidad-estabilidad cuyo contenido deriva de, y subsecuentemente condiciona temporalmente, las actitudes humanas hacia uno mismo y los demás.

En consecuencia, la noción de plan es particularmente robusta y se revela más exigente desde el punto de vista metafísico, cuando es usada para identificar las propiedades necesarias de las entidades sociales. De hecho, una entidad legal es un plan si, y solo si, (1) es el resultado de un proceso orientado a un propósito, (2) su estructura es parcial y anidada, (3) tiene la pretensión de resolver la pregunta acerca de cómo actuar y (4) hace que sus destinatarios tengan la disposición a cumplir con ella ${ }^{35}$. Ahora bien, la pregunta es: ¿tienen todas las normas jurídicas estas propiedades específicas? Shapiro sabe bien cuáles son las implicaciones de esta pregunta y diseña la noción de plan con el objetivo de evitar problemas categoriales. Manteniendo la orientación metodológica de este trabajo, quisiera señalar una cuestión no categorial, sino condicional. Mi pregunta es: ¿si las normas jurídicas fueran planes, harían el mismo trabajo que las normas jurídicas actualmente hacen en nuestra vida de todos los días?

Para responder a esta pregunta, vale la pena concentrarse en la supuesta propiedad disposicional de los planes. Según Shapiro, los planes crean una disposición al cumplimiento en el sentido que, quienes han adoptado el plan se ven racionalmente obligados a ejecutarlo ${ }^{36}$.

35 Shapiro, op. cit., p. 279.

${ }^{36}$ Según Shapiro, los planes "no solo son entidades positivas que tienen una estructura anidada, sino que se forman en un proceso que dispone a sus sujetos a obrar en con- 
Por ejemplo, si adopto el plan de escribir este artículo, todas las demás cosas constantes, constituiría un autoboicot no usar todos los medios disponibles para ejecutar el plan, tal como usar mi ordenador portátil, si se acepta que mi ordenador portátil es uno de los mejores medios para tal fin ${ }^{37}$. Para decirlo con otras palabras, si la racionalidad instrumental exige que use mi ordenador portátil, entonces debo usarlo para alcanzar el fin que he planificado.

Bueno, uno podría argumentar que esta conclusión carece de justificación, puesto que no existe una razón evidente para afirmar que los planes implican una obligación de adoptar un determinado medio, para obtener un cierto fin. Según John Broome, por ejemplo, si yo no creo que debo usar mi ordenador portátil para escribir este artículo, aun cuando esto sea lo que debo hacer para alcanzar el fin que he planificado, no estoy racionalmente obligado a usar mi ordenador portátil para escribir este artículo. En efecto, los deseos y la intención que la racionalidad instrumental exige que yo posea, no son aquellos que debo poseer, a menos que ciertas condiciones relativas a mis creencias se vean satisfechas. Al mismo tiempo, es bastante común que la satisfacción de una condición impuesta por la racionalidad instrumental no contribuya a permitirme alcanzar lo que he planificado. E incluso, algunas veces ello impedirá que logre alcanzar el fin que había planificado. Supongamos, por ejemplo, que creo que no debo escribir este trabajo usando mi ordenador portátil porque no funciona muy bien, pero mi creencia es falsa; mi ordenador portátil funciona perfectamente y es el único medio eficaz para el fin que he planeado. Si he de satisfacer las exigencias de la racionalidad instrumental, mi intención será la de no usar el ordenador portátil, y probablemente no lo usaré. Si esto es así, entonces para satisfacer las exigencias de la racionalidad instrumental, no he de hacer lo que debo hacer. En general, cuando la racionalidad exige que haga $M$, donde $M$ es un medio eficaz para un

formidad. En consecuencia, salvo que exista una disposición a observarlas, las normas creadas para guiar la conducta de los miembros de la comunidad no serán planes" (ibidem, p. 227).

37 Shapiro sostiene que la racionalidad instrumental exige a los agentes que adoptan los medios para sus fines sin deliberación ulterior: véase ibidem, p. 165. 
Tras la naturaleza del derecho. Reflexiones sobre...

fin planificado, puede que no sea el caso que yo deba hacer $M$ y puede que sea el caso que yo no deba hacer $M^{38}$. De este modo, si la racionalidad instrumental exige que yo haga $M$, ello no es claramente una razón suficiente ni necesaria para que yo haga $M$. Ello es así puesto que la racionalidad instrumental es normativa, no en el sentido de obligar al agente a adoptar un medio para un cierto fin, sino meramente en el sentido que exige que entre las actitudes proposicionales de los agentes (creencias, deseos, intenciones, etc.) se dé una particular relación de coherencia.

La falta de fuerza motivacional, que caracteriza a la racionalidad instrumental, se vuelve más evidente en el caso de las actividades sociales institucionalizadas, es decir, cuando los planes individuales se ven determinados por una planificadora anónima, como podría ser el caso de un parlamento o de una autoridad administrativa. Incluso si admitimos, en beneficio de la discusión, que los planes instancian genuinas obligaciones cuando funcionan como "normas internas" y guían la deliberación del agente ${ }^{39}$, es todavía un misterio cómo los planes pueden producir en los agentes la disposición al cumplimiento cuando funcionan como "normas externas", es decir, como normas que no intervienen en la deliberación y el razonamiento práctico de sus destinatarios. En esta situación, los planes no son suficientes para motivar el comportamiento individual, puesto que no implican ningún compromiso por parte de los destinatarios de adoptar un determinado medio para alcanzar el fin planificado ${ }^{40}$.

38 Broome, J., "Does Rationality Give Us Reasons?", Philosophical Issues, 15, 2005, pp. 321337. Véase también Wallace, R. J., "Normativity, Commitment, and Instrumental Reason”, Philosophers' Inprint, 1, 2001, pp. 1-26; Smith, M., "Instrumental Desires, Instrumental Rationality", The Aristotelian Society Supplementary Volume, 78, 2004, p. 97 y ss.

39 Bratman, op. cit., p. 109.

${ }^{40}$ Christine Korsgaard y Joseph Raz han propuesto una línea de razonamiento diferente, pero que conduce a conclusiones similares. Desde su punto de vista, no existen razones para perseguir un fin en cuanto tal. De hecho, "una situación en la que no seguimos los medios para nuestros fines puede ser una situación mejor que aquella en la que sí lo hacemos" (Raz, J., "The Myth of Instrumental Rationality", Journal of Ethics and Social Philosophy, 1, 2005, p. 17). El enunciado según el cual debemos hacer $M$, donde $M$ es un medio efectivo para $P$, depende del contenido de $P$. Véase Korsgaard, C. M., 
En definitiva, lo que quiero señalar aquí es que la racionalidad instrumental no parece ser normativa, en el mismo sentido en el que el derecho lo es. Así, los planes no instancian obligaciones de ningún tipo; los planes, en cambio, instancian un compromiso hacia la coherencia medio-fin que permite evaluar el comportamiento humano. En consecuencia, si las normas jurídicas fueran planes, ellas no harían el trabajo que actualmente hacen en la vida de todos los días, es decir, no instanciarían genuinas obligaciones ni serían autoritativas ${ }^{41}$. Para dar cuenta de la normatividad del derecho y para resolver la Paradoja de la autoridad, Shapiro debería agregar algo a los planes, o admitir que los planes supervienen sobre otras entidades normativas más fundamentales.

De hecho, Michael Bratman excluye este tipo de explicación. Según Bratman, la razón instrumental posee una "base más profunda", a saber, "las estructuras de planificación intertemporal e interpersonal son, en parte, constitutivas de [...] formas de integridad intertemporal y de formas de autogobierno y socialidad intertemporales que consideramos altamente valiosos" 42 . Pero si los planes descansaran sobre valores morales tales como la integridad, el autogobierno y la sociabilidad intertemporal, entonces algunas de las tesis centrales de Legalidad deberían ser modificadas. Según la versión que Shapiro ofrece de la Social Fact Thesis, la existencia de un plan compartido no depende de la existencia de ningún hecho moral ${ }^{43}$. Por ejemplo, los planes fundamentales de un sistema jurídico pueden ser injustos o abyectos y no contar con el apoyo de la población; sin embargo, "si la mayoría de los funcionarios

\footnotetext{
"The Normativity of Instrumental Reason", en Cullity, G. y Gaut, B. (eds.), Ethics and Practical Reason, Oxford, Oxford University Press, 1997, pp. 215-254. En particular, una meta adquiere relevancia normativa solo si (a) vale la pena, y (b) es efectivamente una meta contingente del agente. De ello se sigue que tener un plan de obtener un fin no es suficiente para seguir los medios necesarios para obtener ese fin.

${ }^{41}$ Contra, sin embargo, Bratman, M., Structures of Agency, Oxford, Oxford University Press, 2007, p. 195 y ss.

42 Bratman, M., "Intention, Belief, Practical, Theoretical", en Robertson, S. (ed.), Spheres of Reason. New Essays in the Philosophy of Normativity, Oxford, Oxford University Press, 2009 , p. 56, las cursivas son mías.

43 Shapiro, op. cit., p. 224.
} 
Tras la naturaleza del derecho. Reflexiones sobre...

acepta un plan públicamente accesible y diseñado para ellos, entonces existirá el plan compartido"44. Pero si la tesis de Bratman acerca de la existencia de una "base más profunda" es correcta, entonces esta afirmación de Shapiro debería ser modificada del siguiente modo: no es suficiente que la mayoría de los funcionarios acepte el plan maestro de un sistema jurídico para que ese plan exista. Es necesario que el plan aceptado satisfaga los valores de la integridad, del autogobierno y de la sociabilidad intertemporales. Esto no significa que un sistema jurídico no pueda ser injusto o abyecto. Pero la existencia de planes jurídicos dependerá simplemente de su capacidad para realizar, al menos, aquellos valores humanos que hacen que los planes impongan obligaciones y que aseguran su función de coordinación, incluso si el contenido de la planificación social es injusto o siniestro.

Esta condición ulterior, para la existencia de la planificación jurídica, modificaría significativamente el cuadro de la relación entre derecho y moral ofrecido por Shapiro. Desde el punto de vista de Shapiro, el derecho está necesariamente conectado con la moral, en el sentido de que los planes jurídicos son un tipo de tecnología social, que permite a los seres humanos resolver problemas morales que no podrían resolver de otra manera, aun cuando no sea necesario que los funcionarios se aboquen a consideraciones morales para determinar el contenido del derecho. Sin embargo, si se asume que la tesis de Bratman acerca de las bases más profundas es correcta, se sigue que los planes jurídicos inmorales existen si, y solo si, desempeñan al menos las funciones morales impuestas por los valores de la integridad, el autogobierno y la sociabilidad. En el caso de los planes jurídicos inmorales, los funcionarios tampoco deben abocarse a consideraciones morales para determinar el contenido del derecho, pero si no desempeñan las funciones morales básicas, entonces los planes no son ya aptos para crear obligaciones y por lo tanto no son ni siquiera planes. Si esto es así, el cuadro de Shapiro acerca de la relación entre derecho y moral se acerca sorpresivamente a la idea de "la moral interna del derecho" defendida por Fuller, con la diferencia que, en Legalidad, el carácter orientado a propósitos 
del sistema jurídico se ve garantizado por el principio de racionalidad instrumental. Sobre la base de este análisis la teoría del derecho como plan no puede, tal como en cambio pretendería Shapiro, ser vista como una mera versión actualizada del positivismo jurídico. Shapiro intenta, de hecho, superar la distinción tradicional entre positivismo jurídico y teoría del derecho natural ${ }^{45}$ construyendo un cuadro de la naturaleza del derecho en el que la legalidad y la moral están necesariamente relacionadas, el derecho inmoral es posible, y el contenido del derecho no puede ser concebido independientemente de algunos fines morales.

El punto que he intentado señalar aquí puede ser resumido como sigue. Un primer desafío para el proyecto de Shapiro consiste en especificar cuáles son las bases más profundas de los planes y cómo estos poseen aptitud para generar obligaciones. Este desafío podría conducir a Shapiro a precisar (o incluso modificar) algunas de las tesis centrales presentadas en Legalidad. De acuerdo con el propósito de este trabajo, sin embargo no será necesario intentar establecer con mayores detalles cómo esta tarea podría ser realizada. Debemos en cambio analizar las razones que han llevado a Shapiro a proponer un cuadro de la naturaleza del derecho que posee este problema. ¿Cuál es el recorrido metodológico que subyace a la idea según la cual las normas jurídicas son planes o normas similares a planes? Una respuesta parcial a esta pregunta puede encontrarse en el segundo compromiso del método teórico-jurídico usado por Shapiro, a saber, el compromiso con una explicación constructivista del derecho.

45 Según la distinción tradicional entre positivismo jurídico y derecho natural, este último afirma que el contenido de las leyes depende necesariamente de lo que exige la moral, mientras que el primero no. De acuerdo con esta imagen tradicional, se sigue que para el derecho natural la ley inmoral es imposible, mientras que ello no es así para el positivismo jurídico. Véase sobre esto Coleman, J., "The Architecture of Jurisprudence", The Yale Law Journal, 121, 2011, pp. 2-80. Shapiro efectivamente supera esta distinción al sostener que la ley inmoral es posible y que el contenido de las leyes necesariamente depende de lo que exige la moral. 
Tras la naturaleza del derecho. Reflexiones sobre...

\section{Constructivismo}

Como hemos visto, Shapiro sostiene que una de las técnicas más útiles para el análisis conceptual es la "estrategia constructivista". Para descubrir la naturaleza del derecho, se puede construir un sistema jurídico hipotético a partir de una situación no jurídica, y especificar aquello que es necesario agregar a esa situación no jurídica para transformarla en un sistema jurídico. Las ventajas de esta estrategia son tres, afirma Shapiro. Primero, "permite a los filósofos descartar aquellas propiedades que son características meramente contingentes de la legalidad". De este modo, la estrategia permite a los filósofos descubrir las propiedades necesarias del derecho y desarrollar un análisis no circular de ellas ${ }^{46}$. Shapiro observa que esta es la estrategia usada por el mismo Hart en El concepto de derecho. La situación del asaltante es el punto de partida no jurídico de una explicación de la naturaleza del derecho que avanza marcando la distinción entre estar obligado y tener una obligación, y concluye analizando la estructura del sistema jurídico y la naturaleza de la regla de reconocimiento. Shapiro parece abrazar la misma estrategia en Legalidad. Su punto de partida es la situación no jurídica del plan de cocinar junto a un amigo, avanza mediante el análisis de los dispositivos organizativos que han de ser agregados para que la planificación sea posible, con relación a grupos cada vez más grandes, hasta llegar a la planificación de actividades compartidas de carácter masivo y altamente institucionalizadas como el derecho.

Si bien las estrategias constructivistas de Hart y Shapiro son parecidas, no son idénticas. Existe una importante diferencia entre ellas. En el relato de Hart, la situación del asaltante es aquello que el derecho no puede ser. En el relato de Shapiro, planear cocinar en conjunto con un amigo es aquello que todavía no es derecho. Esta última forma de actividad social posee el genotipo funcional de toda forma de actividad compartida (incluidas las actividades jurídicas), mientras que el cuadro del asaltante de Hart no las posee. Al mismo tiempo, las condiciones agregadas por Hart a la situación de partida ayudan a resaltar, de un

46 Shapiro, op. cit., pp. 48-49. 
modo crítico, por qué el derecho no puede ser reducido a la amenaza de un asaltante. Por el contrario, las condiciones que Shapiro agrega buscan demostrar que el derecho puede ser visto como reflejando in crescendo la actividad de cocinar en conjunto con un amigo.

Desde un punto de vista metodológico, esto es posible gracias al hecho que los planes son usados para identificar una regularidad, recursiva, en el modo en que funciona la guía del comportamiento humano. Esto permite al análisis conceptual explicar la naturaleza de un fenómeno social masivo, reconstruyéndolo como un conjunto de regularidades funcionales cada vez más complejas, pero que poseen idénticas propiedades funcionales ${ }^{47}$. Para lograr esto, el análisis conceptual procede de la siguiente manera: cada uno de los conceptos jurídicos relevantes -tales como OBLIGACIÓN, CONTRATO, PROPIEDAD, LEGISLATURA, SISTEMA JURÍDICO, AUTORIDAD, etc.- es explicado mediante la identificación de las condiciones bajo las cuales la planificación social produce las mismas situaciones que ordinariamente cubre el concepto a explicar. Por ejemplo, para explicar el concepto SISTEMA JURÍDICO es necesario determinar, en primer lugar, cuáles son los problemas que la planificación social enfrenta en aquellas situaciones que ordinariamente cubre el concepto sISTEMA JURÍDICO. Y luego, en segundo lugar, es necesario especificar bajo qué condiciones la planificación social resuelve esos mismos problemas y, de ese modo, vuelve verdadero el concepto ordinario. Así, la noción de plan funciona como un mecanismo recursivo que calcula, tal como lo hace la máquina de Turing, qué es necesario para que una determinada función sea satisfecha en un dominio diferente e incrementalmente mayor.

Las ventajas de este tipo de explicación son obvias. Evita la circularidad y el regreso al infinito, dos dificultades que tradicionalmente ha tenido que sortear el análisis conceptual. Asimismo, el problema del fundamento del derecho, un problema que por lo general se considera ha de resolver una investigación sobre la naturaleza del derecho, resulta

47 Por Shapiro, "las tecnologías de la planificación, incluso las más complejas que son activadas por el derecho, pueden ser construidas mediante la agencia humana exclusivamente" (Ibidem, p. 202). 
Tras la naturaleza del derecho. Reflexiones sobre...

excluido de la agenda filosófica, puesto que la esencia del derecho puede ser explicada ahora en términos meramente funcionales. Este tipo de explicación genera, sin embargo, algunos inconvenientes. De hecho, el análisis teórico-jurídico se limita ahora al estudio de aquellos aspectos del fenómeno jurídico, o aquellas propiedades de una entidad jurídica determinada, que satisfacen la relación funcional que está en la base del derecho. ¿Asegura esta estrategia la mejor explicación de la naturaleza del derecho? Parecería que ello no es el caso, al menos sobre la base de las siguientes dos razones.

En primer lugar, el método usado por Shapiro para construir su teoría consiste en proyectar las propiedades de un microfenómeno, como la planificación individual, sobre un macrofenómeno masivo, como el derecho, lo que acarrea el riesgo de una generalización apresurada. Los macrofenómenos sociales no poseen necesariamente las mismas propiedades constitutivas que los microfenómenos sociales. Considérese, por ejemplo, nuestra concepción estándar de legislatura. Según la teoría del derecho como plan, la legislatura es una institución jurídica en la que un grupo de criaturas planificadoras planifican para otros, lo que permite la coordinación y el control social. Ahora bien, ¿la planificación legislativa, posee las mismas propiedades constitutivas que la planificación individual? Como hemos visto, la panificación individual involucra actitudes de intención caracterizadas por la consistencia y la coherencia medio-fin. Un ser humano es un agente planificador solo si asume el compromiso de cumplir con esas reglas de racionalidad. Por el contrario, legislar no involucra necesariamente tales actitudes. Las leyes suelen ser el resultado irracional de coaliciones efimeras y de agendas políticas arbitrarias. Aún más, el contenido del derecho no puede ser reconducido a las intenciones de las personas que lo crearon, puede ser solo en el sentido débil de que los legisladores tenían la intención de crear derecho, pero no en el sentido fuerte de que todos los legisladores tenían la intención de crear la misma ley ${ }^{48}$. En este sentido, lo que comúnmente denominados "intención del legislador" no posee las mismas propiedades constitutivas que posee la intención individual.

48 Véase Raz, J., Between Authority and Interpretation, op. cit., p. 265 y ss. 
En segundo lugar, el método que Shapiro usa para la construcción teórica tiende a proyectar las propiedades necesarias y relevantes de los macrofenómenos apenas mencionados sobre todos los subfenómenos que equivalen a este. De este modo, es posible que la teoría del derecho como plan no capture las propiedades relevantes de una determinada entidad o de un determinado fenómeno jurídicos, i. e., qué es lo que hace que esa entidad o fenómeno sea lo que es y qué lo distingue de otras entidades o fenómenos. Si ello es correcto, entonces los conceptos jurídicos así diseñados no darían cuenta de ciertas afirmaciones sobre la entidad conceptualizada enraizadas en nuestras asunciones ordinarias acerca del derecho. Algo así sucede si consideramos, por ejemplo, los conceptos de PROPIEDAD y CONTRATO. Para Shapiro, las reglas de la propiedad y los contratos son planes institucionalizados que permiten la planificación privada. Así, afirma que "las reglas sobre propiedad [...] pueden comprenderse como planes generales cuya función es crear las condiciones favorables para que emerja un orden espontáneamente"49. Pero esta descripción no captura algunas propiedades relevantes de las entidades legales. El propietario de una porción de terreno no está obligado a cumplir con un determinado propósito funcional. La concepción estándar de la propiedad inmueble incluye, entre otras cosas el jus abutendi, i. e., "el derecho de destruir o dañar [lo que nos pertenece], si así lo deseamos" 50 . Igualmente, el contratante tiene la facultad de asumir una obligación aun si no maximiza sus intereses desde el punto de vista de la racionalidad instrumental. En este sentido, la propiedad inmueble y los contratos parecen ser expresión de un modelo normativo de acción que no puede ser reducido a la planificación, o que no son planes en el mismo sentido funcional en el que, por ejemplo, el derecho antitrust lo es.

${ }^{49}$ Shapiro, op. cit., p. 176.

${ }^{50}$ Pound, R., "The Law of Property and Recent Justice", American Bar Association Journal, 25, 1939, p. 997. Una discusión crítica del "derecho a extinguir" en el derecho angloamericano puede verse en McCaffery, E. J., "Must We Have the Right to Waste?", in Munzer, S. R. (ed.), New Essays in the Legal and Political Theory of Property, Cambridge, Cambridge University Press, 2001, pp. 76-105; Penner, J. E., "“The Bundle of Rights' Picture of Property”, UCLA Law Review, 43, 1996, pp. 711-820. 
Tras la naturaleza del derecho. Reflexiones sobre...

Brevemente, me parece que la estrategia constructivista de Shapiro posee un carácter recursivo que tiende a confundir entidades legales heterogéneas, i. e., que tiende a reducirlas a un único genotipo funcional que oscurece sus diferencias. La estrategia constructivista de Hart, por el contrario, es sobre todo disyuntiva. Se concentra en aquello que distingue fenómenos tales como estar obligado y tener una obligación, las regularidades sociales y las reglas sociales, la obligación jurídica y la obligación moral, las reglas primarias y las reglas secundarias, etc. De este modo, Hart implícitamente propone un cuadro ontológico más rico y mejor articulado, sobre el que regresaré en la última sección de este artículo.

Obviamente, podría alegarse que esta objeción no da en el blanco. La teoría del derecho consiste en una investigación sobre las propiedades esenciales de las entidades jurídicas que han de ser descubiertas a través del análisis conceptual. No se preocupa por las propiedades contingentes de las legislaturas, los contratos o los derechos de propiedad. Esta respuesta es sin embargo errónea. Si se concibe el análisis conceptual como un tipo de inferencia a la mejor explicación, las propiedades en cuestión han de ser no solo necesarias, sino también interesantes. Para dar un ejemplo de esto, uno podría explicar la explosión de un auto-bomba en el centro de Kabul en términos de las propiedades físicas necesarias de las moléculas individuales que determinaron la explosión, pero esto no sería una explicación interesante del evento en todo ámbito de discurso. Shapiro incluso admite que la relevancia de una propiedad depende del contexto ${ }^{51}$. Pero, desde su punto de vista, que esos criterios dependan del contexto no implica que la naturaleza del derecho dependa del contexto. De acuerdo con el esquema metafísico de Legalidad, existe un, y solo un, conjunto de propiedades relevantes que identifican a las entidades legales en todo mundo posible. La

51 "Que los filósofos consideren interesante una determinada propiedad necesaria dependerá, en alguna medida, del contexto específico: dependerá de qué cuestiones y fenómenos parecen más desconcertantes en un momento dado. Cada generación identifica nuevos problemas, y estos nuevos desafíos centrales inciden en las propiedades que los filósofos del derecho intentarán catalogar y estudiar" (Shapiro, op. cit., p. 35). 
teoría del derecho podría fracasar en descubrirlas, pero esto no afecta el modo en que el mundo realmente es.

Numerosas preguntas pueden ser formuladas respecto de esta reconstrucción crítica de la estrategia que Shapiro propone para construir una teoría del derecho. En cuanto concierne a nuestro propósito aquí, vale la pena concentrarse nuevamente en la fuente de los problemas metodológicos previamente señalados. De hecho, si se mira más atentamente, se advierte que las inconsistencias entre la explicación micro y la macro, que pueden surgir durante la construcción de una teoría, dependen del modo en el que se analizan los fenómenos sociales. Esto dirige nuestra atención hacia el análisis conceptual y el original modo en el que es concebido en Legalidad.

\section{VI. ¿Qué semántica para el análisis conceptual?}

Shapiro sostiene que el análisis conceptual constituye una empresa metafísica. El análisis conceptual permite a la teoría del derecho identificar las condiciones de identidad y las propiedades necesarias de las entidades jurídicas. ¿De qué modo?

Hemos visto que el análisis conceptual se desarrolla en dos etapas. Primero, se reconstruye nuestra concepción ordinaria de los hechos centrales del derecho, lo que permite aislar los casos en los que es posible que el derecho exista. Segundo, estos casos posibles son divididos entre aquellos en los que los hechos específicos acerca del derecho tienen lugar y aquellos en los que no. Esto se lleva a cabo mediante la construcción teórica. Según Shapiro, la teoría del derecho como plan identifica las condiciones necesarias para la producción de los casos posibles y ofrece la mejor explicación de este hecho básico.

Los teóricos del derecho familiarizados con el análisis conceptual seguramente poseerán una preocupación preliminar respecto de esta asunción. De acuerdo con el modelo estándar de análisis, la necesidad conceptual desborda la necesidad metafísica, puesto que una determinada afirmación puede ser conceptualmente verdadera y, al mismo tiempo y bajo la misma descripción, ser metafísicamente falsa. 
Tras la naturaleza del derecho. Reflexiones sobre...

Es conceptualmente verdadero que las brujas están poseídas por el demonio pero esto no es metafísicamente verdadero, puesto que esto no es así en todo mundo posible, y deberíamos estar agradecidos por ello. Pero, ¿bajo qué condiciones, entonces, la necesidad conceptual se corresponde con la necesidad metafísica?

Para resolver esta cuestión pueden ser usadas dos estrategias que, a su vez, se corresponden con dos enfoques diferentes de la ontología social. De acuerdo con la primera estrategia, la estructura de la realidad refleja, en algún modo, la estructura de nuestros conceptos. Este es el caso, por ejemplo, del cuadro que John Searle presenta de las instituciones sociales y de las entidades jurídicas. Un cuadro basado en las reglas constitutivas y en la intencionalidad colectiva ${ }^{52}$. Desde esta perspectiva, una afirmación acerca de la naturaleza del derecho es verdadera o falsa, pero no es metafísicamente objetiva. El tipo de necesidad que este enfoque ofrece es epistémico y asume, además, que existe una discontinuidad ontológica entre los hechos empíricos o brutos y los hechos institucionales, algo que no encaja bien con el proyecto de Shapiro.

De acuerdo con la segunda estrategia, por el contrario, nuestros conceptos reflejan, de algún modo, la estructura de la realidad. En otras palabras, una verdad conceptual acerca de los contratos identifica una propiedad necesaria de los contratos solo si el concepto CONTRATO, y aquellos contratos con los que nos topamos en la vida diaria, poseen esa misa estructura profunda. La idea general que subyace a esta estrategia es que tanto los conceptos jurídicos como los hechos jurídicos son entidades complejas, y que los constituyentes últimos de los conceptos se corresponden con los constituyentes últimos de los hechos, en el sentido que estos últimos determinan la verdad de los primeros. Si, de hecho, esto no fuera así, entonces sería un misterio cómo es que logramos salir adelante en nuestra vida diaria, i.e., cómo es que logramos responder exitosamente a los estímulos externos, transmitir información verdadera a los demás, satisfacer nuestras necesidades contrayendo matrimonio o comprando una casa, etc. La interacción entre seres humanos, la comunicación lingüística, las prácticas socia-

52 Véase Searle, J., The Construction of Social Reality, New York, Free Press, 1995. 
les y las instituciones jurídicas funcionarían por arte de magia. Por lo tanto, a menos que existan algunas razones específicas en contra, podemos asumir que la estructura de nuestro pensamiento y de nuestro lenguaje refleja categorías ontológicas genuinas, o sea, que en cierto grado esa estructura es la que es en virtud de la estructura del mundo.

Si todo esto es cierto, entonces hay buenas noticias para los filósofos del derecho que aún tienen estima por al análisis conceptual. Desde este punto de vista, las condiciones de referencia de nuestros conceptos son analíticas. Así, la teoría del derecho es capaz todavía de descubrir la naturaleza del derecho mediante, casi únicamente, una reflexión cuidadosa y no tanto mediante la observación. Y puede hacer esto dado que las asunciones de sentido común expresadas en las verdades obvias sobre el derecho revelan la estructura profunda de lo que hay ahí afuera.

Para construir este tipo de explicación no alcanza, sin embargo, con enumerar todas nuestras intuiciones acerca del derecho y ofrecer una teoría que explique las condiciones que determinan qué concepto se corresponde con cuáles hechos. Es necesario además afrontar el problema relativo a cómo los conceptos jurídicos pueden ser acerca de cosas, propiedades y relaciones. Esto es, el análisis conceptual ha de afrontar el problema semántico, i. e., ha de mostrar cómo son posibles las relaciones de correspondencia. Ahora bien, con relación a este aspecto, el enfoque semántico asumido por la metodología de Shapiro me parece insatisfactorio en virtud de las dos razones siguientes:

(1) Semántica superficial e indeterminación. Nuestras asunciones de sentido común acerca de los casos posibles de derecho no son necesariamente verdaderas $^{53}$. Shapiro correctamente observa que, hasta cierto punto, podemos equivocarnos acerca de cuáles son las verdades autoevidentes respecto de una entidad jurídica. Asimismo, las personas pueden razonar de modo falaz, ignorar evidencia relevante, carecer de imaginación, hacerse ilusiones, y todo esto da lugar a desacuerdos entre ellas respecto de las propiedades necesarias del derecho ${ }^{54}$. Pero existe un problema más serio, un problema que puede llevar a los filósofos del derecho a cometer un error sistemático. Las verdades obvias acerca del derecho podrían expresar sim-

${ }^{54}$ Shapiro, op. cit., p. 42 y ss. 
Tras la naturaleza del derecho. Reflexiones sobre...

ples similitudes superficiales entre juegos de lenguaje. Palabras tales como "juez", "parlamento", "regla", "derecho", etc., son ambiguas y vagas; se usan en sentidos bastante diferentes en ámbitos espacio-temporales diferentes, y admiten casos fronterizos que a veces no es fácil resolver. Por lo tanto, no es obvio cómo el análisis de enunciados que contienen estos términos-conceptos vagos y ambiguos, y cuyos sentido y referencia cambian radicalmente en el espacio y en el tiempo, puedan ser de ayuda para construir una lista de las propiedades esenciales de las entidades jurídicas.

(2) Relevancia. El método constructivista de Shapiro persigue aislar las propiedades necesarias y relevantes (o interesantes) de las entidades jurídicas mediante el análisis conceptual. Sin embargo, no resulta muy claro cómo se satisface la condición de relevancia. Si la relevancia es determinada de manera holística, i. e., observando los aspectos sociales y culturales de un fenómeno jurídico sin recurrir a hipótesis analíticas, Shapiro se dirige hacia el relativismo ontológico ${ }^{55}$, que no encaja bien con su concepción de la pregunta de la identidad (volveré sobre esto en la última sección de este artículo). Si la condición de relevancia se satisface sobre la base de cómo es el mundo, el método de Shapiro para la investigación jurídico-filosófica parece descansar en una forma de conocimiento que no depende de la experiencia. Desde este punto de vista, las verdades relevantes y necesarias acerca de la naturaleza del derecho pueden ser derivadas a priori, mediante el análisis conceptual, a partir de verdades que son a posteriori y contingentes, como las verdades obvias acerca del derecho. Está bien lejos de ser obvio, sin embargo, cómo ello puede ser llevado a cabo.

(3) Redundancia del análisis. El enfoque de Shapiro respecto de la teoría del derecho corre el riesgo de cometer el "el error de leer el mundo como si poseyera las propiedades del lenguaje" ${ }^{26}$. Si asumimos que una tesis acerca de la naturaleza del derecho se correlaciona con una entidad jurídica a través de convenciones lingüísticas, entonces esa tesis no refleja necesariamente la estructura interna de la realidad social. Por lo tanto, es posible que una imagen de la naturaleza del derecho que se corresponde

55 Véase Quine, Word and Object, op. cit., pp. 58 y 77.

56 Austin, J., "Truth", Aristotelian Society Supplementary Volume, 24, 1950, p. 129. 
con la teoría del derecho de sentido común no sea una imagen fiable, y que dé una imagen del derecho que meramente refleja nuestros prejuicios, deseos, valores, en lugar de reflejar lo que el derecho realmente es. Ahora bien, en esta discusión, el hecho de que el metafísico no tenga a disposición un punto de vista ontológico independiente del lenguaje es relevante por razones metodológicas. Una vez que un esquema lingüístico es convencionalmente aceptado como ontología, y una vez que se acepta también que no existe una correlación necesaria entre la estructura del lenguaje y la estructura de la realidad, entonces las cuestiones ontológicas pueden ser respondidas simplemente mediante la investigación empírica. Si las premisas apenas mencionadas son verdaderas, el análisis conceptual no tiene nada para decir a la metafísica y a la filosofía en general.

(4) Ceguera semántica. Que exista una correspondencia entre la teoría del derecho de sentido común y la realidad social, tal como asume Shapiro, no es algo que pueda ser constatado, y ello no es algo bueno para la filosofía. Vale la pena advertir, a este respecto, que el enfoque metafísico de Shapiro con relación a la naturaleza del derecho es condicional, no categorial. Nos dice cuál es la naturaleza del derecho si la correspondencia señalada existe. Pero Legalidad no ofrece ninguna evidencia de que esta condición se vea satisfecha, puesto que esto no es una tarea para el análisis conceptual. Desde un punto de vista semántico, esto me parece una posición teórica bastante similar al punto alcanzado por Wittgenstein al final del Tractatus, donde propone una concepción de la relación entre el lenguaje y el mundo que es inaccesible para nosotros. $\mathrm{Al}$ igual que el primer Wittgenstein, Shapiro parece sostener la idea de que las estructuras lingüísticas reflejan estructuras ontológicas a través de una cartografía semántico-conceptual, pero cómo es que esto sucede es algo que no puede ser explicado y ha de ser asumido como cierto. Tal como sugiere Wittgenstein en el Tractatus, Shapiro no se preocupa acerca de esta cuestión y "arroja la escalera, una vez que ha subido por ella" 57 . Todo lo que necesitamos saber es que la teoría del derecho como plan parece funcionar bastante bien. Desde un punto de vista filosófico,

57 Wittgenstein, L., Tractatus Logico-Philosophicus, London-New York, Routledge \& Kegan Paul, 1961, 6.54. 
Tras la naturaleza del derecho. Reflexiones sobre...

sin embargo, la solución de la escalera no es satisfactoria, tal como el mismo Wittgenstein ha argumentado. E incluso, esquivar este problema nos lleva a una metodología completamente diferente para la metafísica, el análisis conceptual y la teoría del derecho.

\section{La pregunta de la identidad y el pluralismo ontológico}

La tesis que intentaré defender en este artículo es que los problemas metodológicos que hemos considerado hasta aquí dependen del modo en que Scott Shapiro ha diseñado la pregunta de la identidad. La exagerada extensión de la teoría de los planes, el reduccionismo en la construcción teórica y la ceguera semántica poseen todos la misma fuente. Descansan en el modo de concebir la pregunta "¿qué es el derecho?". En esta última sección intentaré mostrar por qué ello es así y ofreceré una posible vía de salida. Al hacer esto, no ofreceré una mirada diferente de la pregunta de la identidad, sino que simplemente esbozaré al respecto una línea de razonamiento diferente, también estimulada por el modo en que Shapiro diseña esa pregunta. Como se verá, lo que sigue será también parte de la discusión filosófica del trabajo de Shapiro y no constituye, ni pretende hacerlo, una perspectiva filosófica autónoma.

Como hemos visto en la sección previa, desde el punto de vista de Shapiro la pregunta de la identidad es la pregunta central que ha de responder una investigación acerca de la naturaleza del derecho. Esto es perfectamente consistente con la metafísica contemporánea, en la que se considera que los predicados de existencia expresan típicamente relaciones de identidad, siguiendo las ideas de Quine al respecto. Por ejemplo, cuando una abogada afirma que "el contrato existe" está en realidad afirmando que "es el caso que existe una cosa y esta cosa es idéntica a un contrato" 58 . El problema es que las condiciones bajo las

${ }^{58}$ Es famoso el argumento de Quine según el cual "tenemos una noción bastante aceptable de clase, o de objeto o atributo físico, o de cualquier otro tipo, sólo en tanto y en cuanto tenemos un principio aceptable de identificación de esa clase de objetos. No hay entidades sin identidad". Quine, W.V.O., Theories and Things, Cambridge (Mass.), Harvard University Press, 1981, p. 102. 
cuales las relaciones de identidad se verifican no son obvias. Existen numerosas sendas filosóficas para abordar esta cuestión. Las identidades à la Locke típicamente dependen de la ubicación espacio-temporal de los objetos. Así, si $x$ e $y$ se encuentran en el mismo lugar en el mismo momento, entonces $x=y$. Las identidades mereológicas dependen de cómo las partes se relacionan con el todo. Así, si $x$ e $y$ se componen de las mismas partes, entonces $x=y$. A su vez, las identidades à la Leibniz dependen de las características cualitativas de los objetos. Así, si $x$ e $y$ poseen las mismas propiedades, entonces $x=y$. Shapiro hace suya la última, es decir, la concepción racionalista de las relaciones de identidad. Tal como hemos visto, para "exponer un conjunto de propiedades que hacen de las instancias (reales o posibles) de X el objeto que son" es necesario dar una respuesta satisfactoria a la pregunta de la identidad (véase la sección 2).

¿Cuáles son las consecuencias que se siguen de concebir de este modo la pregunta de la identidad? Brevemente, se asume que las entidades jurídicas consisten en un único universo de hechos. Estos hechos poseen el mismo conjunto de propiedades básicas y el mismo modo de existencia, por lo que resultan cubiertas por un único cuantificador. Este conjunto de propiedades unifica las normas, las prácticas y las instituciones jurídicas, como también nuestras actitudes y nuestro discurso acerca del derecho. Esta asunción básica justifica, a su vez, la idea de que existe una correspondencia entre las asunciones del sentido común acerca del derecho y la realidad jurídica, justifica la explicación de los hechos jurídicos mediante una función recursiva que se ve satisfecha en todos los ámbitos jurídicos, y justifica la tesis según la cual el derecho es una actividad que genera genuinas obligaciones.

Ahora bien, ¿es esta la mejor manera de dar cuenta del problema relativo a la identidad de las entidades jurídicas? Seguramente no es el único. Quisiera explorar una línea alternativa de razonamiento, que sigue las pistas ofrecidas por el modo en que Geach ha abordado el problema de la identidad ${ }^{59}$. Para Geach, los enunciados de identidad que poseen la forma " $\mathrm{x}=\mathrm{y}$ " son incompletos. Si digo que $x$ es mejor que $y$, esto no tiene 
Tras la naturaleza del derecho. Reflexiones sobre...

ningún sentido si no agrego que $x$ es mejor que $y$ en términos de fuerza, color, velocidad, gusto, etc. Igualmente, cuando digo que " $x$ es idéntica a $y$ ", se trata de una expresión incompleta que abrevia " $x$ es lo mismo que $y$ ". Y en este caso es necesario agregar a la expresión inicial que $x$ e $y$ son el mismo contrato, la misma ley, el mismo ministro, el mismo Estado, etc. Más precisamente, las relaciones de identidad dependen del dominio de valores sobre el que se extiende el alcance del cuantificador existencial. Esta tesis ha sido comúnmente interpretada como un tipo de relativismo ontológico. En el discurso filosófico-jurídico, esta tesis implicaría que el derecho como tal no existe; lo que cuenta como derecho depende del dominio metafísico seleccionado por el observador no comprometido, y en consecuencia depende de los hechos e intereses no jurídicos involucrados en la investigación teórico-jurídica. Pero la tesis de Geach podría ser reconstruida de un modo distinto. Una entidad legal está constituida por conjuntos diferentes de propiedades pertenecientes a diferentes dominios de discurso ${ }^{60}$. Si esto es así, entonces términos tales como "ley", "contrato", "propiedad", etc., sufren de una múltiple ambigüedad, a saber, se refieren a conjuntos diferentes de propiedades cuando son aplicados a discursos pertenecientes a dominios diferentes. De esto se sigue que, por ejemplo, las normas poseen un determinado conjunto de propiedades en el dominio de la moral, pero poseen un conjunto diferente en el dominio de la religión, en el dominio del derecho, como también, hasta cierto punto, en el dominio de la ley de contratos, de propiedad, de responsabilidad penal, etc., según cuál sea el uso del término "norma" en cada uno de esos dominios. Desde esta perspectiva, por lo tanto, los criterios de identidad de una entidad jurídica reflejan una irreducible pluralidad de tipos de discursos, i. e., de múltiples juegos de lenguaje en los cuáles se considera que tal entidad posee determinadas propiedades.

Según este tipo de "pluralismo ontológico", por lo tanto, una entidad social como una norma jurídica existe en más de un modo y

${ }^{60}$ Un dominio del discurso (o esquema lingüístico) está constituido por el conjunto de términos de un lenguaje y el conjunto de reglas que gobiernan su uso. La posición deflacionista de la ontología defendida aquí fue inicialmente formulada en Carnap, R., "Empiricism, Semantics and Ontology", Revue Internationale de Philosophie, 4, 1950, pp. 20-40. 
(probablemente) no se encuentra disponible un principio general que unifique los distintos modos de ser de las entidades jurídicas. Además, el pluralismo ontológico considera que la pregunta de la identidad es una pregunta acerca de los usos del lenguaje que instancian un determinado esquema lingüístico, y no como una doctrina metafísica. El monismo de Shapiro afirma que cada uno de estos dominios autónomos de hechos sociales es en realidad parte, junto a los demás, de un único universo o de una única totalidad metafisica, que puede ser descubierta analizando el lenguaje ordinario. Por el contrario, el pluralismo ontológico niega, antes que nada, la unidad del lenguaje. El pluralismo ontológico sostiene que los diferentes usos del lenguaje comparten solo sus propiedades superficiales -que pueden ser capturadas, por ejemplo, observando las verdades obvias acerca del derecho- pero que son fundamentalmente distintos. Al mismo tiempo, todas las afirmaciones ordinarias de existencia referidas a entidades jurídicas están circunscriptas a un dominio del discurso, que constituye el alcance del cuantificador existencial ${ }^{61}$.

Por supuesto, el pluralismo ontológico ha de enfrentar sus propios problemas metodológicos. Entre otras cosas, y sobre la base del "principio de economía", ha de determinar cuáles son los límites de "tolerancia pluralista" para evitar que el número de entidades jurídicas aumente más allá de lo necesario ${ }^{62}$. Asimismo, ha de proporcionar los criterios para identificar cuáles son los dominios de discurso relevantes, junto con una explicación de cómo se superponen y encajan entre ellos. El pluralismo de discurso posee, sin embargo, sus propias ventajas metodológicas. A diferencia del monismo metafísico, implícitamente suscripto por Shapiro, no padece de ceguera semántica. Ello en cuanto la base, de toda explicación ontológica de la realidad, es proveída por un

${ }^{61}$ Véase McDaniel, K., "Ways of Being", en Chalmers, D., Manley, D. y Wasserman, R. (eds.), Metametaphysics: New Essays of the Foundation of Ontology, Oxford, Clarendon Press, 2009, pp. 1290-319; Hirsh, E., "Quantifier Variance and Realism", Philosophical Issues, 12, 2002, pp. 51-73; Price, H., "Metaphysical Pluralism", The Journal of Philosophy, 89, 1992, pp. 387-409.

62 Para una solución del problema, véase Turner, J., "Ontological Pluralism”, The Journal of Philosophy, 107, 2010, pp. 28 y ss. 
Tras la naturaleza del derecho. Reflexiones sobre...

esquema lingüístico que fija las propiedades necesarias y relevantes de una entidad jurídica. Asimismo, una versión pluralista de la pregunta de la identidad no incurre en el reduccionismo del constructivismo teórico. Un conjunto de propiedades necesarias es descripto como una función de múltiples dominios del discurso que dan cuenta del contexto en el que se considera existe una entidad jurídica. Las leyes, los testamentos, los contratos, los tribunales, los parlamentos, todos poseen propiedades necesarias diferentes bajo diferentes descripciones en diferentes dominios del discurso. Finalmente, el pluralismo ontológico no necesita recurrir a un mecanismo recursivo unificador, como los planes, para dar cuenta de todos los aspectos de la realidad jurídica. Sobre la base de este enfoque, el sobrecompromiso explicativo que afecta a la teoría del derecho como plan puede ser evitado. La planificación social identifica las propiedades necesarias de las normas jurídicas en determinados dominios del discurso. En particular, se trata de los dominios en los que la racionalidad instrumental gobierna las prácticas lingüísticas que involucran normas jurídicas, según ciertas necesidades y propósitos sociales. Pero la planificación social no da cuenta de las propiedades necesarias de las normas jurídicas en todos los dominios. Da cuenta de algunos aspectos relevantes de la realidad social e institucional desde un punto de vista no privilegiado. Sobre la base de esto, el pluralismo ontológico podría producir un esquema prometedor para investigar la naturaleza del derecho. Aceptar este esquema llevaría a la teoría del derecho a abandonar algunas de sus tesis universalistas, pero esto debería ser una ventaja en tanto en cuanto permitiera a los filósofos del derecho alcanzar un análisis más fino de la naturaleza de las entidades jurídicas.Para terminar, estoy de acuerdo con Shapiro en que el mundo social es "sumamente plural"63. Pero esta circunstancia admite diferentes explicaciones. El mundo social podría ser altamente pluralista debido a que existe un único universo de hechos sociales si bien altamente diferenciado y articulado, tal como sostiene Shapiro. O, en cambio, podría ser el caso que el mundo social sea altamente pluralista debido a que existen múltiples universos de hechos, propiedades y discursos sociales, que son consistentes y están

${ }^{63}$ Shapiro, op. cit., p. 36. 
ontológicamente determinados, y de los cuales depende la naturaleza del derecho.

\section{Bibliografía}

Austin, J., "Truth", Aristotelian Society Supplementary Volume, 24, 1950, pp. 111-128.

Canale, D., "Consequences of Pragmatic Conceptualism. On the Methodology Problem in Jurisprudence", Ratio Juris, 22, 2009, pp. 171-186.

Carnap, R., "Empiricism, Semantics and Ontology", Revue Internationale de Philosophie, 4, 1950, pp. 20-40.

Coleman, J. L., "Naturalized Jurisprudence and Naturalized Epistemology", Philosophical Topics, 29, 2001, pp. 113-128.

Coleman, J.L., "The Architecture of Jurisprudence", The Yale Law Journal, 121, 2011, pp. 2-80.

Bentham, J., Rationale of Judicial Evidence, Vol. 3, London, Hunt \& Clarke, 1827.

Bratman, M. E., Intention, Plans, and Practical Reason, Cambridge (Mass.), Harvard University Press, 1987.

Bratman, M. E., Structures of Agency. Essays, Oxford, Oxford University Press, 2007.

Bratman, M. E., "Intention, Belief, Practical, Theoretical", en Robertson, S. (ed.), Spheres of Reason. New Essays in the Philosophy of Normativity, Oxford, Oxford University Press, 2009, pp. 29-61.

Broome, J., "Does Rationality Give Us Reasons?", Philosophical Issues, 15, 2005, pp. 321-337.

Dickson, J., Evaluation and Legal Theory, Oxford-Portland, Hart, 2001. Enoch, D., "Reasons-Giving and the Law", en Green, L. y Leiter, B. (eds.), Oxford Studies in Philosophy of Law, Vol. 1, Oxford, Oxford University Press, 2011.

Finnis, J., Natural Law and Natural Rights. Second edition, Oxford, Oxford University Press, 2011.

Fuller, L. L., The Morality of Law. Revised edition, New Haven-London, 
Tras la naturaleza del derecho. Reflexiones sobre...

Yale University Press, 1969.

Geach, P. T., "Identity", Journal of Metaphysics, 21, 1967, pp. 3-12.

Greenberg, M., "How Facts Make Law," en Hershovitz, S. (ed.),

Exploring Law's Empire: The Jurisprudence of Ronald Dworkin, Oxford, Oxford University Press, 2006.

Greenberg, M., "Naturalism in Epistemology and the Philosophy of Law", Law \& Philosophy, 30, 2011, pp. 419-451.

Harman, G. H., "The Inference to the Best Explanation", The Philosophical Review, 74, 1965, pp. 88-95.

Himma, K. E., "Reconsidering a Dogma: Conceptual Analysis, the Naturalistic Turn, and Legal Philosophy", en Freeman, M. y Harrison, R. (eds.), Law and Philosophy: Current Legal Issues, Oxford, Oxford University Press, 2007.

Hirsch, E., "Quantifier Variance and Realism", Philosophical Issues, 12, 2002, pp. 51-73.

Jackson, F., From Metaphysics to Ethics. A Defense of Conceptual Analysis, Oxford, Clarendon Press, 1998.

Kelsen, H., General Theory of Law and State, Cambridge (Mass.), Harvard University Press, 1945.

Korsgaard, C. M., "The Normativity of Instrumental Reason", en Cullity, G. y Gaut, B. (eds.), Ethics and Practical Reason, Oxford, Oxford University Press, 1997, pp. 215-254.

Leiter, B., Naturalizing Jurisprudence. Essays on American Legal Realism and Naturalism in Legal Philosophy, Oxford, Oxford University Press, 2007. McCaffery, E. J., "Must We Have the Right to Waste?", en Munzer, S. R. (ed.), New Essays in the Legal and Political Theory of Property, Cambridge, Cambridge University Press, 2001, pp. 76-105.

McDaniel, K., "Ways of Being", en Chalmers, D., Manley, D. y Wasserman, R. (eds.), Metametaphysics: New Essays of the Foundation of Ontology, Oxford, Clarendon Press, 2009, pp. 1290-319.

Oberdiek, J. y Patterson, D., "Moral Evaluation and Conceptual Analysis in Jurisprudential Methodology", en Freeman, M. y Harrison, R. (eds.), Law and Philosophy: Current Legal Issues, Oxford, Oxford University Press, 2007, pp. 60-75. 
Penner, J. E., "“The Bundle of Rights' Picture of Property”, UCLA Law Review, 43, 1996, pp. 711-820.

Pound, R., "The Law of Property and Recent Justice", American Bar Association Journal, 25, 1939, pp. 993-998.

Price, H., "Metaphysical Pluralism", The Journal of Philosophy, 89, 1992, pp. 387-409.

Quine, W. V. O., Word and Object, Cambridge (Mass.), MIT Press, 1960.

Quine, W. V. O., "On Carnap's Views on Ontology", en Id., The Ways of Paradox and Other Essays, New York, Random House, 1966.

Quine, W. V. O., Theories and Things, Cambridge (Mass.), Harvard University Press, 1981.

Ramsey, F., Philosophical Papers, Cambridge, Cambridge University Press, 1990.

Raz, J., Ethics in the Public Domain: Essays on the Morality of Law and Politics, Oxford, Clarendon Press, 1994.

Raz, J., "The Myth of Instrumental Rationality", Journal of Ethics and Social Philosophy, 1, 2005, pp. 1-28.

Raz, J., Between Authority and Interpretation: On the Theory of Law and Practical Reason, Oxford, Oxford University Press, 2009.

Schauer, F., "The Best Laid Plan", Yale Law Journal, 120, 2011, pp. 586-621.

Searle, J., The Construction of Social Reality, New York, Free Press, 1995. Shapiro, S., Legalidad, Madrid-Barcelona, Marcial Pons, 2014, traducción de Papayannis, D. M. y Ramírez Ludeña, L.

Smith, M., "Instrumental Desires, Instrumental Rationality", The Aristotelian Society Supplementary Volume, 78, 2004, pp. 93-109.

Turner, J., "Ontological Pluralism", The Journal of Philosophy, 107, 2010, pp. 5-34.

Wallace, R. J., "Normativity, Commitment, and Instrumental Reason", Philosophers' Inprint, 1, 2001, pp. 1-26.

Wittgenstein, L., Tractatus Logico-Philosophicus, London-New York, Routledge \& Kegan Paul, 1961. 